\title{
Role of spirometry for preoperative evaluation of stable COPD patients before elective laparoscopic cholecystectomy
}

\author{
Anil Kumar Kodavala ${ }^{1, *}$, Krishna Chaitnanya ${ }^{2}$, Surekha Tadisetti ${ }^{3}$, Vignan Kumar G. ${ }^{4}$ \\ ${ }^{\mathbf{1}}$ Assistant Professor, Dept. of Respiratory Medicine, ${ }^{\mathbf{2}}$ Associate Professor, Dept. of Anaesthesiology, ${ }^{\mathbf{3}}$ Associate Professor, Dept. \\ of Obstetrics and Gynecology, ${ }^{4}$ Professor, Dept. of Respiratory Medicine, Narayana Medical College, Nellore, Andhra Pradesh, \\ India
}

*Corresponding Author:

Email: anil.kodavala@gmail.com

\begin{abstract}
Introduction: Postoperative pulmonary complications (PPC) are the main source of postoperative morbidity and mortality. Chronic Obstructive pulmonary Disease (COPD) patients are at 3 times more risk for the development of PPCs. Spirometry is commonly done as a part of preoperative evaluation. Our objective is to assess role of spirometry to predict PPCs in patients with stable COPD following laparoscopic Cholecystectomy.

Material and Methods: This is a prospective observational study at a tertiary care centre. The study was approved by institutional ethics committee. 134 stable COPD patients posted for elective laparoscopic cholecystectomy under combined general epidural anaesthesia were included. Detailed clinical history was taken, physical examination was done, quality of life was assessed by COPD Assessment Test (CAT) score, chest X-ray, ABG, ECG, 2 D Echo, Spirometry, 6 minute walk test (6MWT) were done. Patients were followed up till discharge. Data of was analyzed by SPSS 11.

Results: Out of 134 patients $112(83.6 \%)$ were males and $22(16.4 \%)$ were females. All were of more than 40yrs. All patients had CAT score $>10.68$ males and 16 females had FEV1 (Forced expiratory volume in 1 second) $<50 \%$ predicted. 10 male patients had hypercapnia. Only 3 male patients developed postoperative pulmonary complications (PPC). Conclusion: In patients with stable COPD of known severity, preoperative spirometry may not provide additional information in predicting PPC. Routine spirometry and arterial blood gases are not required for preoperative pulmonary evaluation in patients with stable COPD prior to laparoscopic cholecystectomy.
\end{abstract}

Keywords: Preoperative; Spirometry; Pulmonary disease; Chronic obstructive; Postoperative complications.

\section{Introduction:}

Postoperative pulmonary complications (PPC) are the main source of postoperative morbidity and mortality. They include postoperative respiratory infection, pneumonia, respiratory failure, bronchospasm, atelectasis, or hypoxemia [1]. The incidence of postoperative pulmonary complications in a general surgical population is around 5\%. This figure increases in selected populations of high-risk patients and surgical procedures [2]. Presence of underlying lung disease, particularly COPD, places the patient at 3 times more risk for the development of PPCs [3,4]. 30 day mortality was high in patients with PPC. Identifying patients who are at a high risk of PPCs, however, can at present help us to design individually tailored management approaches [2]. In the field of surgery, preoperative spirometry and arterial blood gases are widely used to select surgical candidates and minimise the occurrence of postoperative pulmonary complications [4].

In our day to day practice we come across many patients with stable COPD being referred for preoperative evaluation for assessing pulmonary risk. There is equivocal evidence about requirement of spirometry for preoperative evaluation. In this study we assessed requirement of routine spirometry for predicting PPCs in patients with stable COPD undergoing elective laparoscopic cholecystectomy.

\section{Material and Methods:}

In this hospital-based prospective observational study at a tertiary care centre, from January2015 to November 2017, 150 COPD patients posted for laparoscopic cholecystectomy surgery under combined general epidural anaesthesia were included. The study was approved by institutional ethics committee. According to GOLD (Global initiative for chronic obstructive lung disease) criteria, COPD diagnosis was confirmed by using FEV1/FVC, FEV1\% predicted and patients with moderate, severe and very severe disease were selected.

\section{Inclusion criteria}

All stable COPD patients posted for laparoscopic cholecystectomy.

\section{Exclusion criteria}

1. COPD patients with acute exacerbations within last 6 weeks.

2. Post bronchodilator reversibility $>12 \%$ and $>200 \mathrm{ml}$.

3. Patients who had contraindications or not able to perform spirometry.

4. H/o Pulmonary tuberculosis.

5. Patients with other diseases affecting respiratory and cardiovascular systems.

6. Recent thoracic or abdominal surgery/eye surgery or retinal detachment.

7. Patients who did not undergo surgery after enrolment.

8. Patients with non pulmonary postoperative complications. 
Informed consent was taken from all subjects and then they were interviewed, demographic characteristics, clinical history was noted, physical examination was done, quality of life was assessed by using COPD Assessment Test (CAT) score. Using spirometer (Micro Quark, Cosmed, Rome, Italy) FEV1/FVC, FEV1, FVC, FEV1 \% predicted, FVC \% predicted, FEF25-75\% were obtained before and after the patient received salbutamol via a metered-dose inhaler. The best flow-volume loop was used in the final analysis of the data. All calculations were performed using automated processors that were interfaced to the measuring instruments. Severity of disease was assessed by FEV1 \% predicted according to GOLD criteria. Other investigations including chest $X$-ray, arterial blood gases (ABG), ECG, 2-D Echo and 6 minute walk test were done and results were documented. Sputum was sent for AFB staining and culture \& sensitivity.

All patients underwent elective laparoscopic cholecystectomy under combined general epidural anaesthesia and they were followed up postoperatively till discharge. Post operative respiratory symptoms like breathlessness, wheeze, respiratory failure, respiratory infection, pneumonia, pleural effusion and pneumothorax were documented.

Room air saturation $<90 \%$ and $\mathrm{PaO} 2<60 \mathrm{~mm}$ of $\mathrm{Hg}$ was considered as respiratory failure. New onset of fever, yellow sputum and breathlessness was considered as respiratory tract infection and presence of fine inspiratory crackles or patch in chest $\mathrm{x}$ ray was considered as pneumonia.

Data was analyzed by SPSS 11 (the Statistical Program for the Social Sciences of the University of Michigan, Microsoft Corporation, Redmond, USA). Continuous variables were expressed as mean \pm standard deviation (SD), and categorical variables were expressed in numbers and percentages. Descriptive measures were taken for continuous variables like age, BMI (Body mass index), smoking score, CAT score, FEV1, FVC (Forced vital capacity), $\mathrm{PaO} 2$ (Arterial partial pressure of oxygen), $\mathrm{PaCO} 2$ (Arterial partial pressure of carbondioxide), $\mathrm{SpO} 2$ (Arterial oxygen saturation), 6MWD (6 Minute walk distance). As only 3 patient developed PPC, we did not check for correlation between severity of airflow obstruction and incidence of PPC.

\section{Results:}

Initially patients recruited were 150 . Of which, 7 developed acute exacerbation of COPD prior to surgery, 5 did not undergo surgery and 4 developed non pulmonary complications postoperatively. After excluding these, remaining patients $(n=134)$ data was analysed. Out of 134 , $112(83.6 \%)$ were males and $22(16.4 \%)$ were females. All were of more than $40 \mathrm{yrs}$ with a mean age of 63.2 years. 80 males $(71.4 \%)$ and all females $(100 \%)$ were from middle socioeconomic class.

The mean BMI was 21.55. 51.78\% males and 9.09\% females had normal BMI. $23.88 \%$ males had under weight and $19.64 \%$ males and $45.45 \%$ females had over weight.
Among males, $62.50 \%$ had a smoking score of $>20$ pack years and $26.78 \%$ had $\leq 20$ pack years and $8.96 \%$ males were never smokers. $69.64 \%$ males were active smokers. $81.81 \%$ females had exposure to second hand tobacco smoke at home.

All patients (100\%) had shortness of breath, $91.1 \%$ had cough, $66.6 \%$ had sputum production at the time of presentation. $52 \%$ patients had systemic hypertension, nearly $32 \%$ had diabetes mellitus and $29 \%$ have more than one co morbid illness.

All patients had COPD assessment test (CAT) score > 10. $67.85 \%$ males and $72.72 \%$ females had baseline $\mathrm{PaO} 2$ between $60-80 \mathrm{~mm} \mathrm{Hg}$ at rest. Mean $\mathrm{PaCO} 2$ of males was $39.58 \pm 3.10$ and that of females was $39.18 \pm 4.26$. Only 10 males $(8.92 \%)$ had hypercapnia at rest.

Mean forced expiratory volume in first second (FEV1) of males was $1.26 \pm 0.47$ litres and that of females was $0.94 \pm 0.29$ litres. Of males, $39.28 \%$ had moderate COPD, $50 \%$ had severe COPD, and $10.71 \%$ had very severe COPD according to GOLD spirometry criteria. Of females, $27.27 \%$ had moderate and $72.72 \%$ had severe COPD. $8.93 \%$ males and $9.09 \%$ females had Pulmonary artery hypertension.

Mean 6 minute walking distance of males was 361.03 \pm 63.89 meters and that of females was $340.18 \pm 59.55$ metres. $89.28 \%$ males and $90.9 \%$ females walked more than 300 meters distance and $10.71 \%$ males and $9.10 \%$ females walked $<300$ meters.

All patients underwent elective laparoscopic cholecystectomy under combined general epidural anaesthesia. Out of 134, 2 had postoperative respiratory failure and only 1 had postoperative Respiratory tract infection. None of the females developed postoperative pulmonary complications. No postoperative mortality.

\section{Discussion:}

There are limited studies in this aspect in our locality and the prediction of risk of postoperative pulmonary complications by spirometry lacks agreement. Various studies reported varying incidence of PPC. But few risk factors were identified in some studies.

Old age was a risk factor for PPC in some studies. Age $\geq 70$ years following CABG surgery in a study by Erik HJ Hulzebos et al, [10] age > 65 years following gastrectomy in a study by Oh Jeong et al,. [9] age $>60$ following laparoscopic gastrectomy in a study by Redondo Ntutumu et al [11], age > 40 yrs in another study by Charlene K Mitchell et al [6]. Avanced age was identified as a risk factor for PPC following emergency abdominal surgery by Livia Goreth Galvão Serejo et al. [12] and after elective upper abdominal surgery by A. Kocabas et al, and Jaume Canet et al. In our study $55.36 \%$ males and $54.55 \%$ females were of the age above 60 years and all were above 40 years. All the 3 patients who had PPC in our study were more than 70 years of age. Wide range of age in study group was a reason for lower incidence of PPC. 
Abnormal BMI was a risk factor for PPC after emergency abdominal surgery in a study by Livia Goreth Galva o'Serejo et al [12]. In our study $28.57 \%$ males and $45.45 \%$ females had underweight. $19.64 \%$ males and $45.45 \%$ females had over weight. Out of 3 patients who had PPC, 2 had over weight and developed postoperative respiratory failure. Abnormal BMI may be a risk factor for PPC even in patients with stable COPD. As majority of study patients had normal BMI, incidence of PPC was low.

Preoperative productive cough was a risk factor for PPC in studies by Erik HJ Hulzebos et al and Charlene K Mitchell et al $[6,10]$. In our study $62.68 \%$ patients had productive cough, but none of them had PPC. Exclusion of patients with acute exacerbation of COPD in last 6 weeks resulted in reduced incidence of PPC in study group.

Smoking was a risk factor for PPC in studies by A Kocabas et al and Erik HJ Hulzebos et al [5,10]. In our study $69.64 \%$ patients were active smokers. But 3 patients developed PPC. Smoking also has variable effect over PPC.

Diabetes mellitus was a risk factor for PPC in a study by Erik HJ Hulzebos et al [10]. In our study 52.23\% patients had Diabetes mellitus, but no one developed PPCs as we excluded patients with uncontrolled blood sugars.

Low preoperative arterial oxygen saturation was a risk factor for PPC in studies by L. FUSO et al [7] Jaume Canet et al [8]. In our study as all subjects had normal preoperative arterial oxygen saturation. Stable COPD patients with normal arterial oxygenation may have reduced incidence of PPC.

Upper abdominal surgery was a risk factor for PPC in studies by Charlene K Mitchell et al and Jaume Canet et al [6,8]. Livia Goreth Galva o'Serejo et al also found upper or upper/lower abdominal incisions was associated with PPC [12]. Even though all the study group (100\%) underwent laparoscopic cholecystectomy (upper abdominal surgery) only $3(2.23 \%)$ patients developed PPC. Laparoscopy associated with low incidence of PPC when compared to open surgeries.
Emergency surgery was a risk factor for PPC in a study by Jaume Canet et al [8]. Our study group underwent elective laparoscopic cholecystectomy. This supports promoting elective surgeries in COPD patients may reduce incidence of PPC.

The abnormal preoperative spirometry i.e, low FEV1 was a risk factor for PPC in studies by A Kocabas et al [5], L. FUSO et al [7], Oh Jeong et al [9], Kimball et al [12]. Preoperative FEV1 was inversely related to incidence of PPC. In our study $60.71 \%$ males and $72.72 \%$ females had $\mathrm{FEV}_{1}<50 \%$ of predicated. Because, presence of spirometric abnormalities alone has limited clinical value as a screening test to predict PPC prior to laparoscopic cholecystectomy.

Pre-existing COPD was a risk factor for PPC in studies by Kimball et al [12], Redondo Ntutumu et al [11], Oh Jeong et al. [9]. In our study all subjects were confirmed and stable COPD. This indicates mere presence of pre-existing lung disease like COPD not a contraindication for surgery if it is well controlled or stable. Excluding/avoiding surgery in patients with acute exacerbation my further reuduces incidence of PPC in COPD.

Longer duration of anaesthesia was a risk factor for PPC in studies by A Kocabas et al [5], Charlene K Mitchell et al [6], Redondo Ntutumu et al [11] and Jaume Canet et al [8]. In our study, average duration of surgery is $2.37 \pm 0.60$ hours. Duration of anaesthesia alone may not predict PPC, as there was no uniform opinion over cut off for duration of surgery/anaesthesia.

There is no standard opinion regarding common pulmonary complications following surgery. Respiratory failure was most common PPC in studies by L Fuso et al [7] (15\%) Jaume Canet et al [8] (2.6\%) In a study by Redondo Ntutumu et al pneumonia, was the common PPC (67.5\%).[11] In our study, 2 patients had respiratory failure and 1 had respiratory infection.

\section{Limitations}

Small sample size.

No control group.

Table 1: Incidence of PPC in various studies

\begin{tabular}{|l|l|c|c|}
\hline \multicolumn{1}{|c|}{ Study } & \multicolumn{1}{c|}{ Nature of surgery } & Sample size (n) & Incidence of PPC \\
\hline A. Kocabas et al [5] & elective upper abdominal surgery & 60 & $35 \%$ \\
\hline Charlene K Mitchell et al [6] & elective non thoracic surgery & 148 & $11 \%$ \\
\hline L. FUSO et al [7] & elective upper abdominal surgery & 480 & $18 \%$ \\
\hline Redondo Ntutumu et al [11] & laparoscopic gastrectomy & 1205 & $6.8 \%$ \\
\hline Oh Jeong et al [9] & $\begin{array}{l}\text { Laparoscopic and open } \\
\text { gastrectomy in patients with } \\
\text { airflow limitation }\end{array}$ & 91 & $6.2 \%$ \\
\hline Erik HJ Hulzebos et al [10] & CABG surgery & 117 & \\
\hline Present study & $\begin{array}{l}\text { Elective laparoscopic } \\
\text { cholecystectomy }\end{array}$ & 134 & $2.24 \%$ \\
\hline
\end{tabular}

PPC: postoperative pulmonary complications 
Table 2: Baseline patient characteristics $(\mathbf{n}=134)$

\begin{tabular}{|l|c|c|}
\hline \multicolumn{1}{|c|}{ Variable } & Mean & $\begin{array}{c}\text { Standard } \\
\text { deviation }\end{array}$ \\
\hline Age & 63.2 & 8.92 \\
\hline BMI & 21.55 & 5.68 \\
\hline Smoking pack years & 21.38 & 9.27 \\
\hline CAT score & 17.14 & 2.31 \\
\hline Duration of symptoms & 6.43 & 3.46 \\
\hline FEV1 & 1.21 & 0.46 \\
\hline FVC & 2.06 & 0.68 \\
\hline PaO2 & 76.10 & 6.48 \\
\hline PaCO2 & 39.52 & 3.28 \\
\hline SpO2 & 95.49 & 1.35 \\
\hline 6MWD & 357.61 & 63.24 \\
\hline Duration of surgery (hours) & 2.37 & 0.60 \\
\hline $\begin{array}{l}\text { Duration of hospital stay } \\
\text { after surgery (days) }\end{array}$ & 3.70 & 1.10 \\
\hline scre-COPD ass & & \\
\hline
\end{tabular}

BMI-Body mass index, CAT score-COPD assessment test score, FEV1-Forced expiratory volume in 1 second, FVC-Forced vital capacity, PaO2- Arterial partial pressure of oxygen, PaCO2- Arterial partial pressure of Carbon dioxide, SpO2- Arterial oxygen saturation, 6MWD-6 minute walk distance.

\section{Conclusion:}

Incidence of PPC depends on many patient related and surgery related factors. Presence of COPD alone may not be a deterring factor for laparoscopic cholecystecomy. Routine preoperative spirometry in patients with stable COPD and known severity may not provide additional information for risk assessment before elective laparoscopic cholecystectomy. Stabilizing or deferring surgery in patients with acute exacerbation of COPD before laparoscopic cholecystectomy further reduces incidence of PPC. Further larger randomised and controlled studies are required to generalise the results.

Conflicts of interest: No potential conflict of interest relevant to this article was reported.

Acknowledgements: The authors thank Dr Rammohan $\mathrm{P}$ for his valuable suggestions.

\section{References:}

1. Canet J, Mazo V.Minerva. Postoperative pulmonary complications. Anestesiologica 2010;76(2):138-43.

2. Canet, Jaumea; Gallart, Lluísb. Predicting postoperative pulmonary complications in the general population. Current Opinion Anaesthesiol 2013;26(2):107-15.

3. Rock P, Passannante A. Preoperative assessment: pulmonary. Anesthesiol Clin N Am 2004;22(1):77-91.

4. William R. Kimball, MD, PhD. The Role of Spirometry in Predicting Pulmonary Complications after Abdominal Surgery: Progressing toward an Answer. Anesthesiol1999;90(2):356-57.

5. A. Kocabas, K. Karat, G. H. SiiNmzt, R. Burgut. Value of preoperative spirometry to predict postoperative pulmonary complications. Respir Med 1996;90:25-33.

6. Mitchell CK, Smoger SH, Pfeifer MP, Vogel RL, Pandit MK, Donnelly PJ etal. Multivariate analysis of factors associated with postoperative pulmonary complications following general elective surgery. Arch Surg 1998;133(2):194-8.
7. L. Fuso, L. Cisternino, A. Dinapoli, V. Dicosmo, L. M. Tramaglino, S. Basso, et al. Role of spirometric and arterial gas data in predicting pulmonary complications after abdominal surgery. Respir Med 2000;94:1171-76.

8. Canet J, Gallart L, Gomar C, Paluzie G, Valles J, Castillo J, et al. Prediction of postoperative pulmonary complications in a population-based surgical cohort. Anesthesiol 2010;113:1338-50.

9. Jeong O, Ryu SY, Park YK. The value of preoperative lung spirometry test for predicting the operative risk in patients undergoing gastric cancer surgery. J Korean Surg Soc 2013;84(1):18-26.

10. Hulzebos EH, Van Meeteren NL, De Bie RA, Dagnelie PC, Helders PJ. Prediction of postoperative pulmonary complications on the basis of preoperative risk factors in patients who had undergone coronary artery bypass graft surgery. Phys Ther 2003;83(1):8-16.

11. Ntutumu R, Liu H, Zhen L, Hu YF, Mou TY, Lin T, I $\mathrm{BA}$, et al. Risk factors for pulmonary complications following aparoscopic gastrectomy: A single-center study. Med (Baltimore) 2016;95(32):e4567.

12. Serejo LG, da Silva-Junior FP, Bastos JP, de Bruin GS, Mota RM, de Bruin PF. Risk factors for pulmonary complications after emergency abdominal surgery. Respir Med 2007;101:808-13.

How to cite this article: Anil Kumar Kodavala A. K., Chaitnanya K., Tadisetti S., G. Vignan K. Role of spirometry for preoperative evaluation of stable COPD patients before elective laparoscopic cholecystectomy. IP Indian J Immunol Respiratory Med 2018;3(3):127-130. 